\title{
Diagnostic Accuracy of the Xpert MTB/RIF Assay for Lymph Node Tuberculosis: A Systematic Review and Meta-Analysis
}

\author{
Guocan Yu, ${ }^{1}$ Fangming Zhong $\left(\mathbb{D},{ }^{1}\right.$ Bo Ye ${ }^{D},{ }^{1}$ Xudong Xu ${ }^{D},{ }^{1}$ \\ Da Chen $\left(\mathbb{1},{ }^{1}\right.$ and Yanqin Shen $\left(\mathbb{D}^{2}\right.$ \\ ${ }^{1}$ Department of Thoracic Surgery, Hangzhou Red Cross Hospital, Hangzhou, Zhejiang, China \\ ${ }^{2}$ Department of Tuberculosis, Hangzhou Red Cross Hospital, Hangzhou, Zhejiang, China \\ Correspondence should be addressed to Yanqin Shen; yanqinshen@yeah.net
}

Received 19 November 2018; Accepted 15 April 2019; Published 19 May 2019

Academic Editor: Isabel Portugal

Copyright (C) 2019 Guocan Yu et al. This is an open access article distributed under the Creative Commons Attribution License, which permits unrestricted use, distribution, and reproduction in any medium, provided the original work is properly cited.

\begin{abstract}
Objectives. To evaluate the performance of Xpert MTB/RIF for lymph node tuberculosis (LNTB). Methods. We searched databases for published reports. We reviewed the studies and identified the performance of Xpert MTB/RIF with respect to a composite reference standard (CRS) and culture. We used a bivariate random-effects model to perform meta-analyses and used metaregression to analyze sources of heterogeneity. Results. 15 independent studies compared Xpert MTB/RIF with CRS while 21 comparing it with culture were included. The pooled sensitivity and specificity of Xpert MTB/RIF were $79 \%$ and $98 \%$ compared to that of CRS, respectively, and $84 \%$ and $91 \%$ compared to that of culture, respectively. The pooled sensitivity and specificity using fine needle aspiration (FNA) samples versus CRS were $80 \%$ and $96 \%$, whereas those against culture were $90 \%$ and $89 \%$, respectively. The percentages while working with tissue samples versus CRS were $76 \%$ and $100 \%$, respectively, whereas those against culture were $76 \%$ and $92 \%$, respectively. There was no significant difference in diagnostic efficiency among the types of specimen. Conclusions. Xpert MTB/RIF demonstrates good diagnostic efficiency for LNTB and is not related to the type of specimen, obtained via different routes.
\end{abstract}

\section{Introduction}

Tuberculosis is one of the most serious challenges to global public health [1]. Besides causing pulmonary tuberculosis (PTB), Mycobacterium tuberculosis infection may spread to extrapulmonary sites, causing extrapulmonary tuberculosis (EPTB), LNTB being its most common type. At present, fine needle aspiration (FNA) of lymph node and biopsy are used for diagnosis, although the accuracy is relatively low, and traditional diagnostic protocols, such as Mycobacterium tuberculosis culture, are quite time-consuming. Mostly, biopsy with histopathological examination and culture are required for proper diagnosis; effective control of tuberculosis lies in its rapid diagnosis and treatment. Therefore, a rapid laboratory diagnosis of tuberculosis is an urgent necessity. The Xpert MTB/RIF assay is a rapid, automated molecular test with high accuracy in PTB and EPTB detection [2]. This assay has also been recommended for the diagnosis of LNTB and has shown good diagnostic efficiency [3]. However, the diagnostic efficiency with different types of lymph node specimens (FNA and tissue samples) remains controversial. Due to lack of independent systematic research of the diagnostic accuracy of Xpert MTB/RIF assay for LNTB, the possibility of influence of type of specimen (FNA and tissue samples) on the results is yet to be clarified. For this purpose, we performed a meta-analysis to confirm the diagnostic performance of Xpert MTB/RIF assay, compared to that of CRS and mycobacterial culture, in the detection of LNTB, using different types of specimen, obtained in different ways from individuals with suspected LNTB. We analyzed the pooled sensitivity and specificity of this assay against different references. Moreover, diagnostic efficiency of the test was evaluated, based on different types and conditions of samples, by subgroup analysis.

\section{Methods}

2.1. Data Sources and Search Strategy. On Jul 6, 2018, we searched PubMed, Embase, the Cochrane Library, China 
National Knowledge Infrastructure (CNKI), and the Wanfang database for studies evaluating the accuracy of Xpert in LNTB detection. The search formula ((Xpert OR Gene Xpert) AND (Tuberculosis, Lymph Node"[Mesh] OR "Extra pulmonary tuberculosis")) was used for PubMed without any limitation. Similar search formulae were used for Embase, the Cochrane Library, CNKI, and Wanfang databases. References cited in the included articles and reviews were further explored for possible candidate studies.

2.2. Inclusion Criteria. We included full-text original studies that assessed the diagnostic accuracy of Xpert assay for LNTB using FNA or biopsy tissue specimens. Reference standards were well-defined and appropriate in the studies. The articles directly provided true positive (TP), false positive (FP), false negative (FN), and true negative (TN) values for the assay or included the data necessary to calculate these measures. Case reports, studies with $<10$ samples, conference reports, and abstracts without full articles were excluded.

2.3. Reference Standard. A composite reference standard (CRS) or mycobacterial culture was defined as the reference standard in our study. Clinical manifestation, biochemical test results, histopathology, smears, other nucleic acid amplification tests (NAATs), culture, and response to antituberculosis treatment constituted the reference standards in CRS.

2.4. Literature Screening and Selection. Two investigators independently assessed the candidate articles by reviewing titles and abstracts, followed by the full text, for inclusion. Discrepancies between the two investigators were resolved by discussion with a third investigator.

2.5. Data Extraction. We extracted data including author, year, country, TP, FP, FN, and TN values for the assay, reference standard, and specimen type, along with other parameters. The same two investigators independently extracted the necessary information from each of the included articles; we cross-checked the information obtained by them. Discrepancies between the two datasets were settled by discussion with a third investigator, similar to that during the literature selection phase. Data from studies against two different reference standards were treated separately.

2.6. Assessment of Study Quality. According to the two reference standards (CRS and culture), the two investigators independently divided the studies into two groups and used a revised tool for Quality Assessment of Diagnostic Accuracy Studies (QUADAS-2) to assess study quality separately [4]. Publication bias was not assessed, since these methods were not applicable to studies of diagnostic accuracy [5].

2.7. Data Synthesis and Statistical Analysis. We first obtained the values corresponding to TP, FP, FN, and TN in each included study and calculated the estimated pooled sensitivity and specificity of Xpert MTB/RIF associated with 95\% CI, against CRS or culture, using bivariate randomeffects models. Forest plots for sensitivity and specificity were generated for each study. The area under summary receiver operating characteristic (SROC) curves (AUC) was subsequently calculated. $\mathrm{I}^{2}$ statistics was used to assess heterogeneity between the studies and a reference standard. While $0 \%$ indicated no observed heterogeneity, values greater than $50 \%$ were considered to signify substantial heterogeneity $[6,7]$. We explored different types of samples, decontamination method, sample conditions, and homogenization as potential sources of heterogeneity, using subgroup and metaregression analyses. At least four published studies were required to carry out the meta-analysis for a predefined variable type. Data from studies against CRS and culture were analyzed separately. Stata version 14.0 (Stata Corp, College Station, TX) with the midas command packages was used to generate forest plots of sensitivity and specificity with 95\% CI for each study and carry out meta-analyses and metaregression analyses.

2.8. Imperfect Reference Standard. Imperfect reference standards may lead to misclassification of samples in diagnostic validity studies $[8,9]$. For the paucibacillary nature of EPTB, a culture would be an imperfect reference standard and lead to an underestimation of the true specificity of Xpert MTB/RIF. A CRS is a composite standard that comprises results from several tests; however, a CRS itself may have reduced specificity, thereby leading to apparent FN Xpert $\mathrm{MTB} / \mathrm{RIF}$ results, an underestimation of the true sensitivity of Xpert MTB/RIF $[9,10]$. Therefore, a study comparing Xpert MTB/RIF with both culture and CRS might provide a more credible range for sensitivity and specificity.

\section{Results}

3.1. Identification of Studies and Study Characteristics. Three hundred and four candidate articles, identified from relevant databases using our search strategy, three articles identified from other sources, and twenty-seven qualified articles were included according to the inclusion criteria (Figure 1) [11-37]. The number of specimens evaluated in each article ranged from 11 to 348 with a median of 118 . Twenty-four articles were written in English and three in Chinese. We excluded one study that had the same data as another included study [38] and five other articles that reported sensitivity only, without reporting any specificity [39-43].

When an article reported the use of two different standards in the same study, we considered the article to include two independent studies. In accordance with this principle, 36 independent studies were included: 15 compared Xpert MTB/RIF with CRS and 21 compared Xpert MTB/RIF with culture (Table 1). Twenty-one studies used FNA samples and 15 used biopsy tissue samples.

3.2. Study Quality. The overall methodological quality of the included studies, using a CRS and culture, is summarized in Figure 2.

\subsection{Diagnostic Accuracy of Xpert MTB/RIF Assay for LNTB} Detection. Fifteen studies included comparison of 1597 FNA or tissue samples with a CRS; Xpert MTB/RIF sensitivity ranged from $49 \%$ (95\% CI 35-63\%) to $97 \%$ (95\% CI 


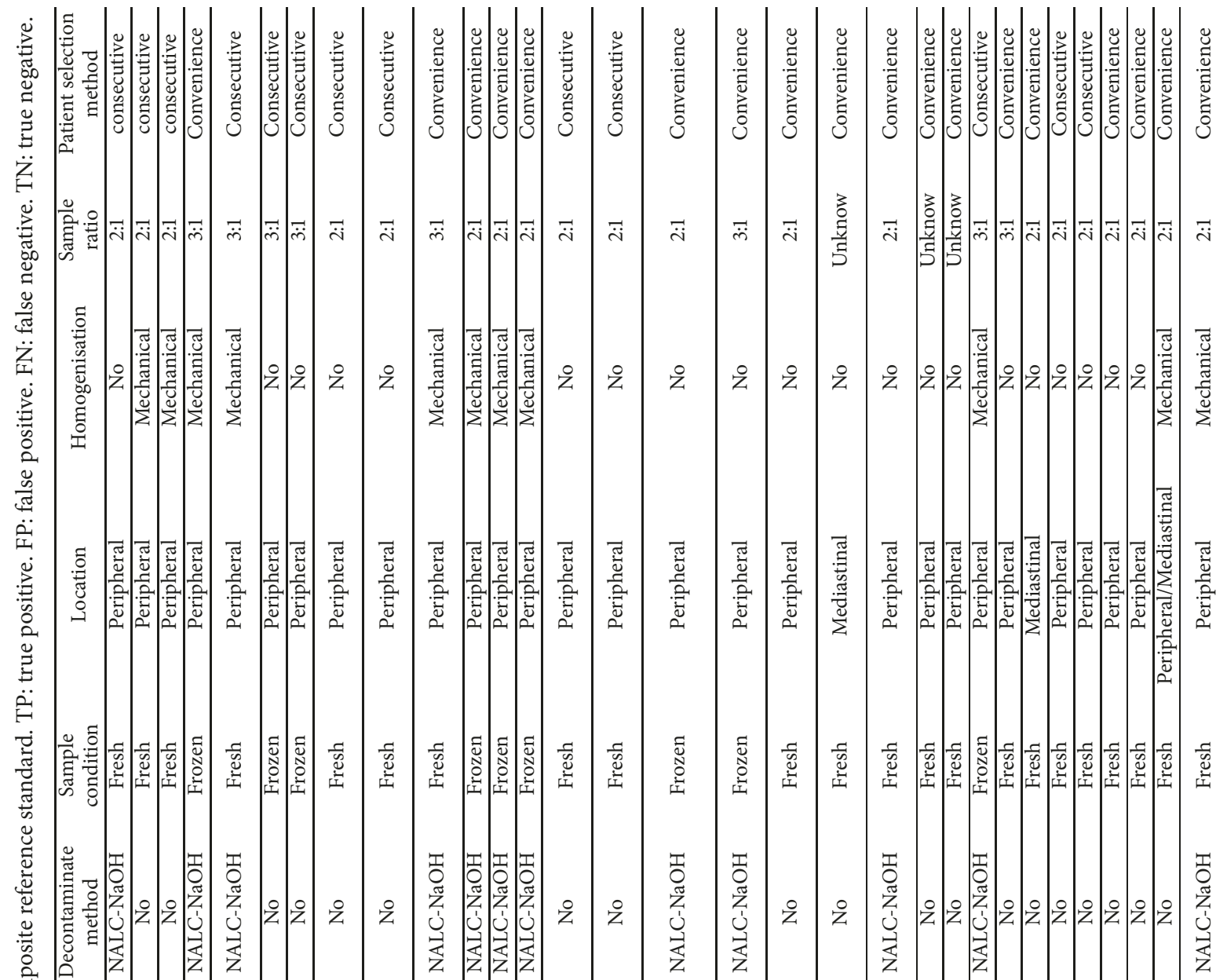

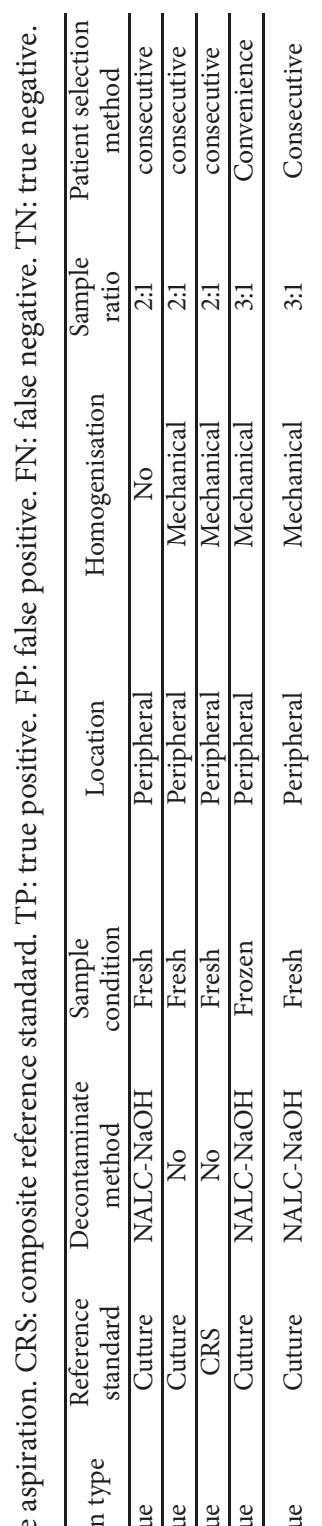

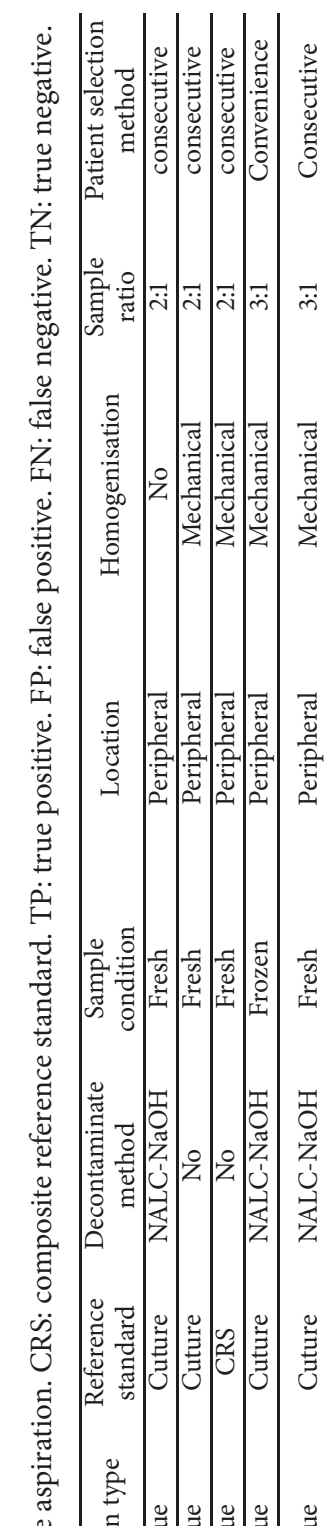

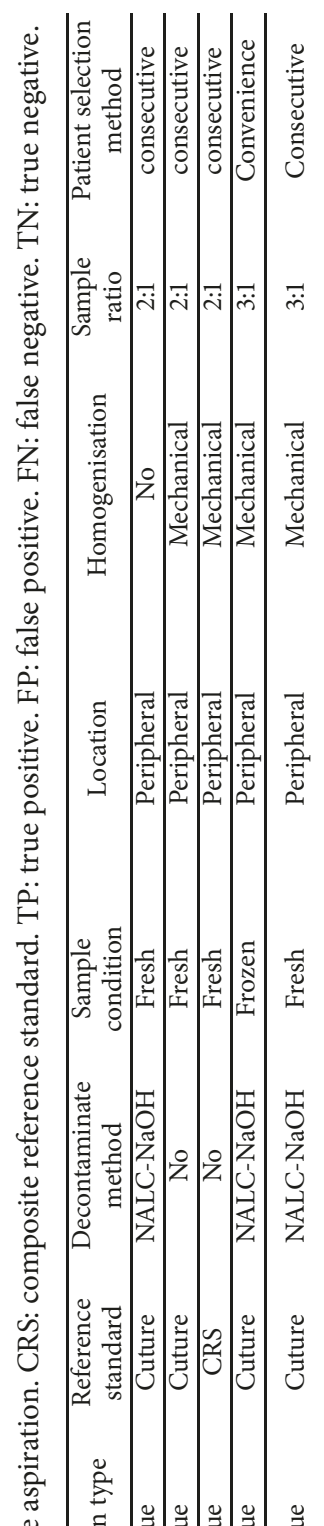

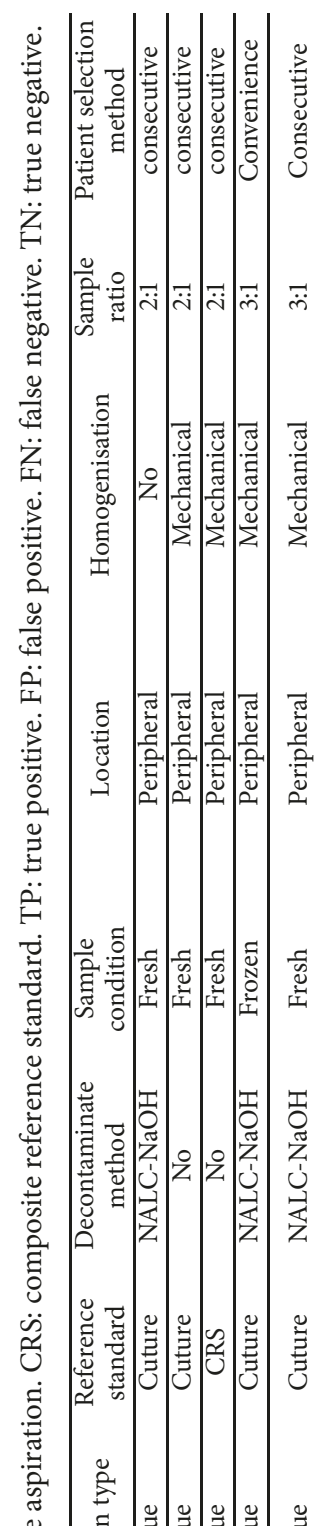

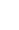

.

,

要

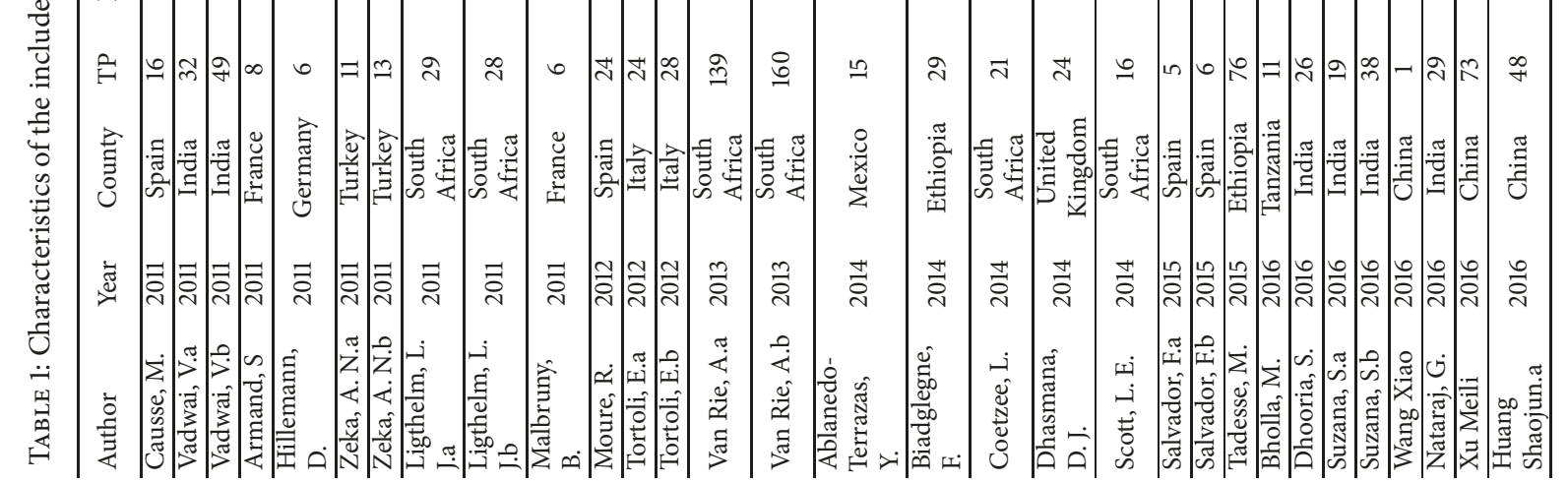




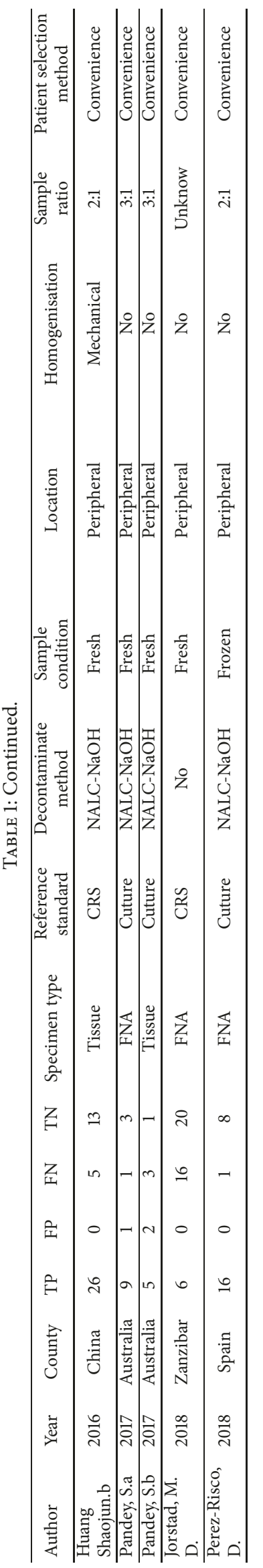



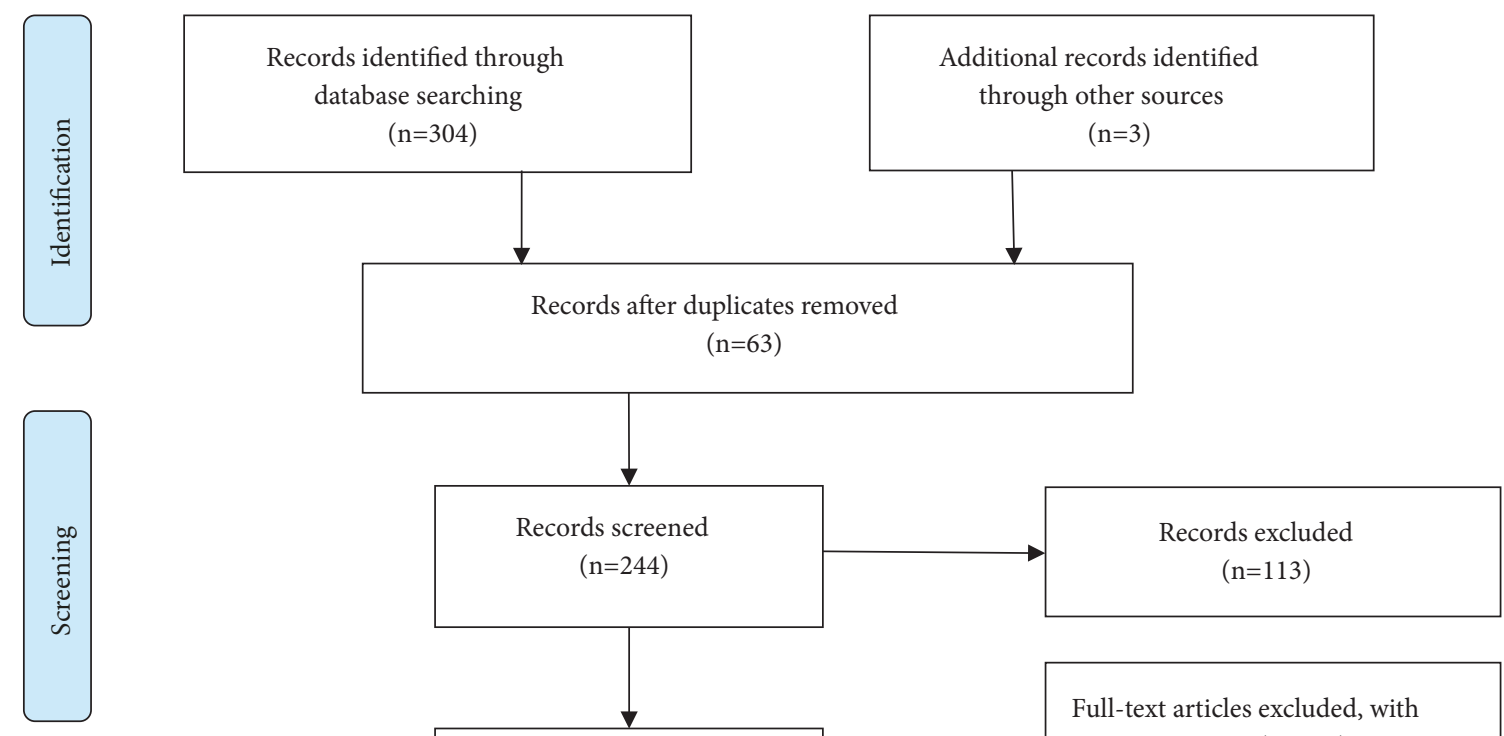

Records after duplicates removed

$$
(n=63)
$$
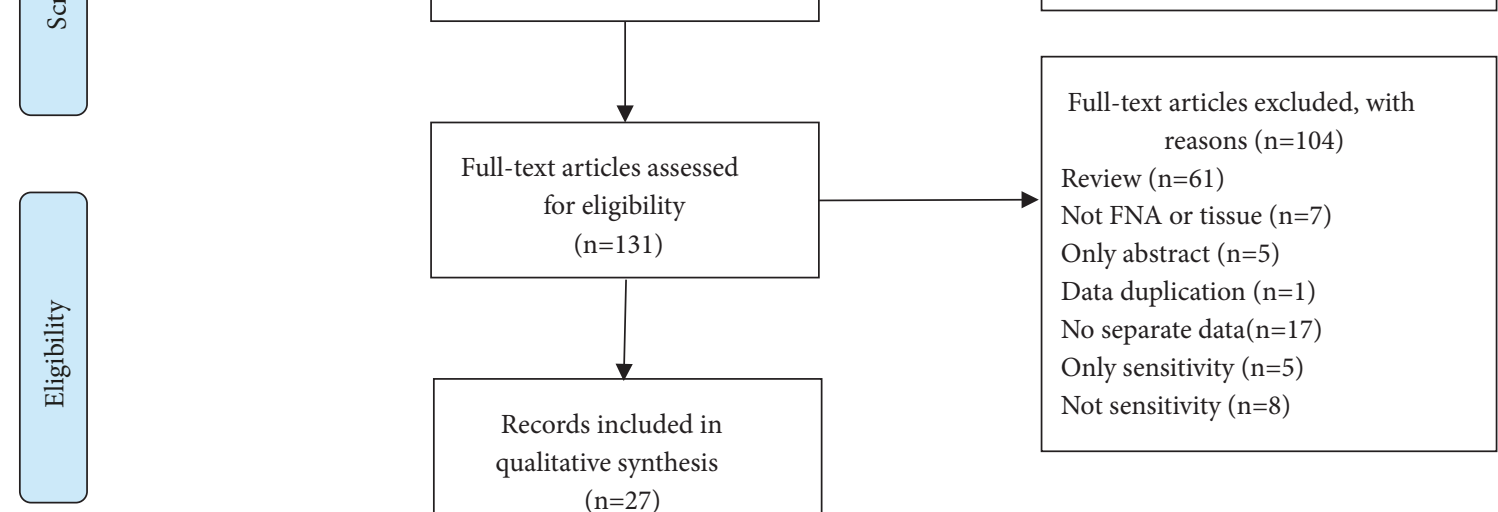

qualitative synthesis

$(n=27)$
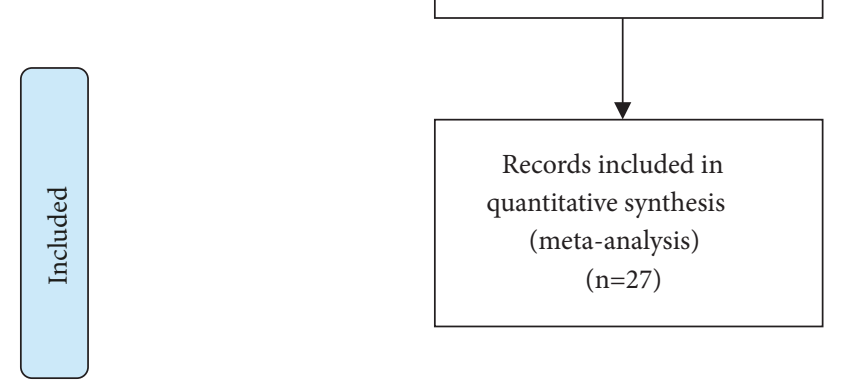

Figure 1: Literature retrieval flow chart. 80, 23, 159, 30, and 12 articles were found from PubMed, the Cochrane Library, Embase, Wanfang database, and CNKI respectively.

83-100\%). Pooled sensitivity of Xpert MTB/RIF assay for LNTB was 79\% (95\% CI 69-86\%) and $\mathrm{I}^{2}$ statistical values were $86 \%$. Xpert MTB/RIF specificity ranged from $72 \%(95 \%$ CI $57-84 \%$ ) to $100 \%$ (95\% CI $97-100 \%)$. Pooled specificity of Xpert MTB/RIF assay was 98\% (95\% CI 94-99\%) and $\mathrm{I}^{2}$ statistical values were $89 \%$ (Figure 3 ). The heterogeneity of sensitivity and specificity was significant. The AUC of SROC was 0.96 (95\% CI 0.94-0.98).

Compared to a culture reference standard, the pooled sensitivity of Xpert MTB/RIF was 84\% (95\% CI 77-90\%) with $\mathrm{I}^{2}=67 \%$ and specificity was $91 \%$ (95\% CI 78-96\%) with $\mathrm{I}^{2}=92 \%$ for 1629 FNA or tissue specimens from 21 studies (Figure 4). The heterogeneity of sensitivity was acceptable; however, that of specificity was significant. The AUC of SROC was 0.92 (95\% CI 0.89-0.94) versus that of culture, suggesting excellent overall diagnostic validity.
We explored the heterogeneity among studies, using subgroup and metaregression analyses on predefined subgroups of sample types, decontamination method, sample condition, and homogenization used in the assay.

In case of Xpert MTB/RIF using FNA samples, sensitivity ranged from $27 \%$ (95\% CI 11-50\%) to $97 \%$ (95\% CI 83-100\%) against a CRS. The pooled sensitivity was $80 \%$ (95\% CI $63-90 \%$ ) with $\mathrm{I}^{2}=90 \%$, and the pooled specificity was 96\% (95\% CI 90-98\%) with $\mathrm{I}^{2}=89 \%$ (Figure 5(a)). There was significant heterogeneity in sensitivity and specificity among studies of Xpert MTB/RIF assay using FNA samples compared to a CRS. The AUC of SROC was 0.96 (95\% CI 0.94-0.97), suggesting very good overall diagnostic validity. When using tissue samples, sensitivity ranged from $68 \%(95 \%$ CI $54-80 \%$ ) to $85 \%$ (95\% CI 68-95\%), and specificity ranged from $99 \%$ (95\% CI $96-10 \%)$ to $100 \%$ (95\% CI $96-100 \%$ ) 


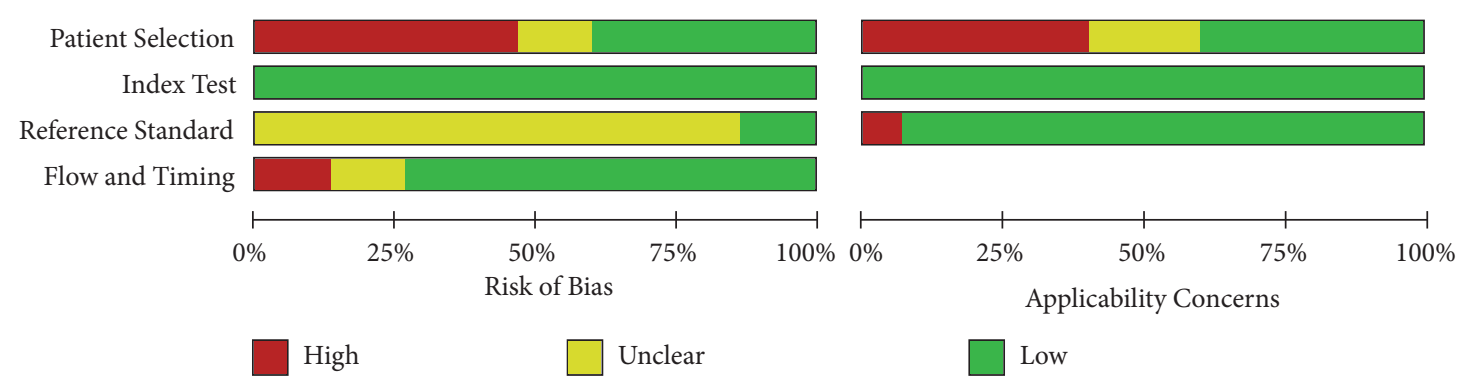

(a)

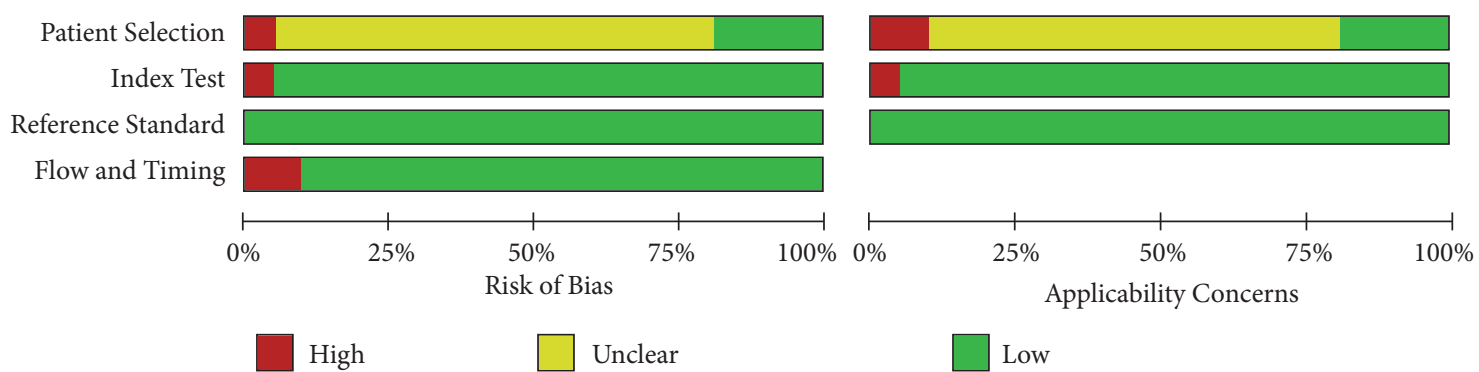

(b)

FIGURE 2: Methodological quality graphs (risk of bias and applicability concerns) as percentages across the included studies. (a) Composite reference standard. (b) Culture reference standard.

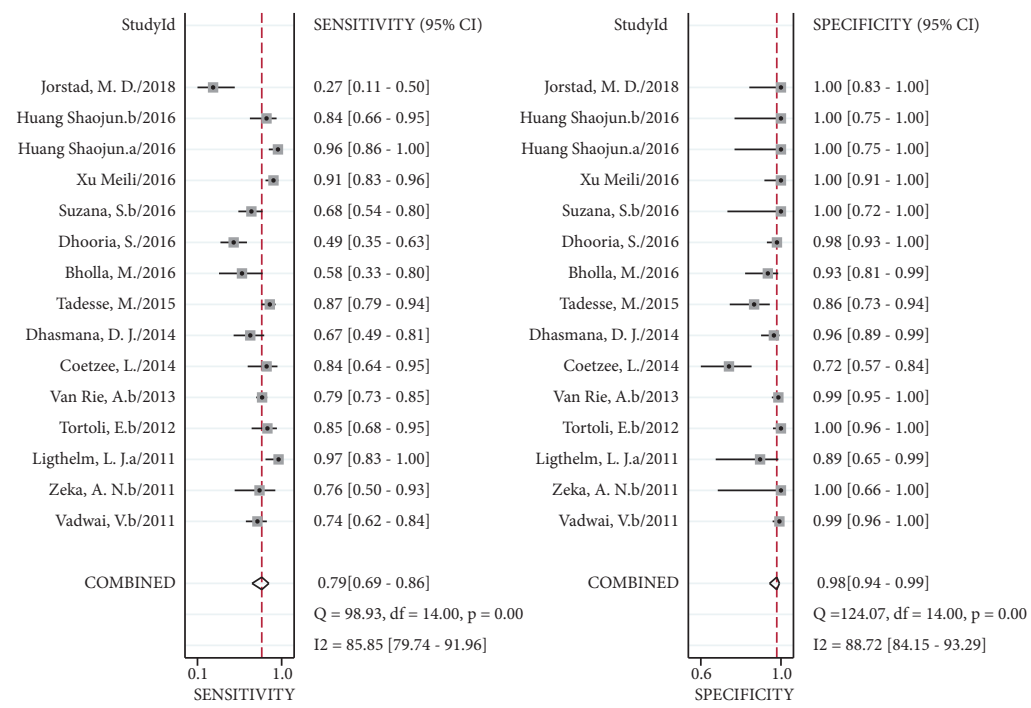

FIgURE 3: Forest plot of Xpert sensitivity and specificity for tuberculosis detection in LNTB compared with a composite reference standard. The squares represent the sensitivity and specificity of a study, and the black line represents their confidence intervals. The diamonds represent the pooled sensitivity and specificity and their confidence intervals. LNTB: lymph nodes tuberculosis.

against a CRS. The pooled sensitivity and specificity of Xpert MTB/RIF assay using tissue samples versus CRS were 76\% (95\% CI 68-82\%) with I2 = 13\%, and 100\% (95\% CI 97-100\%) with $\mathrm{I} 2=0$ (Figure 5(b)). Heterogeneity across studies of Xpert MTB/RIF assay using tissue samples compared to that of CRS was not obvious. The AUC of SROC was 0.96 (95\% CI 0.94-0.98).

In Xpert MTB/RIF using FNA samples, sensitivity ranged from $71 \%(95 \%$ CI $29-96 \%)$ to $100 \%$ (95\% CI $78-100 \%)$ against culture. The pooled sensitivity was 90\% (95\% CI $83-95 \%)$ with $\mathrm{I}^{2}=52 \%$, and pooled specificity was $89 \%(95 \%$ CI 65-97\%) with $\mathrm{I}^{2}=95 \%$ (Figure 6(a)). While heterogeneity of sensitivity was acceptable, there was significant heterogeneity of specificity when using FNA samples, compared to that with culture. The AUC of SROC was 0.94 (95\% CI 0.92-0.96). When using tissue samples, sensitivity ranged from $50 \%$ (95\% CI $25-75 \%)$ to $95 \%$ (95\% CI 75-100\%), and specificity ranged from $33 \%$ (95\% CI 1-91\%) to $100 \%$ (95\% CI 

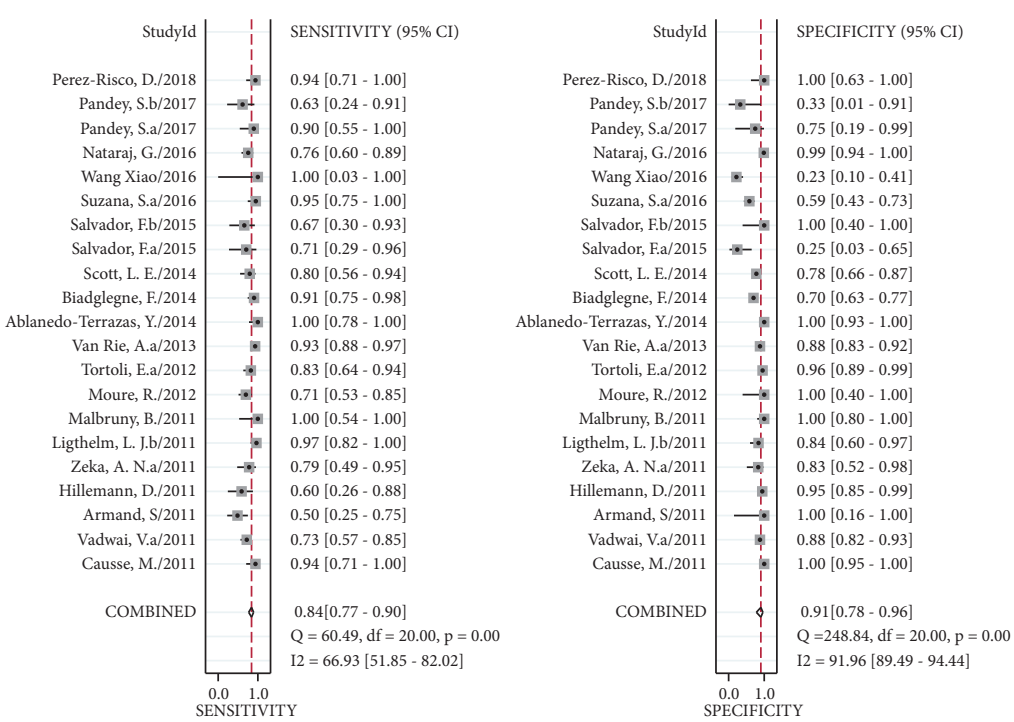

FIGURE 4: Forest plot of Xpert sensitivity and specificity for tuberculosis detection in LNTB compared with culture reference standard. The squares represent the sensitivity and specificity of a study, and the black line represents their confidence intervals. The diamonds represent the pooled sensitivity and specificity and their confidence intervals. LNTB: lymph nodes tuberculosis.

95-100\%) against culture. The pooled sensitivity and specificity of Xpert MTB/RIF assay using tissue samples versus culture were $76 \%$ (95\% CI 66-83\%) with $\mathrm{I}^{2}=49 \%$, and $92 \%$ (95\% CI 78-98\%) with $\mathrm{I}^{2}=86 \%$ (Figure 6(b)). Heterogeneity was very obvious with tissue samples compared to that with culture. The AUC of SROC was 0.86 (95\% CI 0.83-0.89).

Metaregression analysis showed that studies with FNA samples and tissue samples, compared to a CRS, showed similar sensitivity (79\% and $78 \%$, metaregression $\mathrm{P}=0.35)$. However, studies with FNA samples, compared to a CRS, showed lower specificity (96\%) than those using tissue samples (100\%); the difference was statistically significant (metaregression $\mathrm{P}<0.01$ ). The sensitivity of studies using FNA samples was higher than that of studies using tissue samples, compared to culture (90\% versus $76 \%$ ); however, the difference was not statistically significant (meta-regression $\mathrm{P}$ $=0.43$ ). The specificity of studies using FNA samples was lower than that of studies using tissue samples, compared to culture ( $89 \%$ versus $93 \%$ ); this difference was also not statistically significant (metaregression $\mathrm{P}=0.56$ ).

Metaregression analysis showed that decontamination method (with NALC-NaOH or without), sample condition (fresh or frozen), homogenization (mechanical or otherwise), and patient population (high/low income) did not have any effect on Xpert MTB/RIF sensitivity and specificity (metaregression $\mathrm{P}>0.05$ ), compared to that in CRS. These factors were, therefore, obviously not a source of heterogeneity across the studies.

\section{Discussion}

Diagnosis of EPTB, including LNTB, is very challenging, due to the characteristics of a low bacterial load. In most cases, invasive examinations are necessary. For LNTB, the most commonly used invasive procedures are FNA and biopsy. Specimens obtained by different methods have different sensitivity for the diagnosis of LNTB. Sensitivity of pathological diagnosis is the highest with the most invasive biopsy specimens [26]. FNA is widely used in the diagnosis of LNTB, due to its simplicity and safety, but its sensitivity ranges from $10 \%$ to $80 \%$ for a positive mycobacterial culture $[44,45]$. The preferred method of obtaining specimens from patients suspected with LNTB remains controversial. Several published studies had reported positive Ziehl-Neelsen staining and culture to correlate to FNA and biopsy [26, 44]. On the contrary, granulomatous inflammation observed in biopsy specimens was much more frequent than in FNA specimens (96.8\% versus 54.8\%, respectively, $\mathrm{P}<0.001$ ) [26]. This finding was helpful in the diagnosis of Ziehl-Neelsen stain- and culture-negative cases.

Nucleic acid detection has been widely used in the diagnosis of tuberculosis [46]. The Xpert MTB/RIF assay is currently one of the most commonly used nucleic acid detection methods. This test can confirm rifampicin resistance while validating the MTB complex DNA within $2 \mathrm{~h}$. It is widely used for diagnosing PTB and EPTB. Several reports on its diagnostic efficiency have been published [47]. The assay has also been recommended by WHO for the diagnosis of LNTB. Systematic review and meta-analysis published in 2014 and 2015 showed good diagnostic efficiency of Xpert MTB/RIF for LNTB [3, 48]. However, there has been no consensus on the sensitivity of this test for specimens (FNA and biopsy specimens) obtained by different methods. For this purpose, we designed this study to determine the performance of Xpert MTB/RIF in FNA and biopsy specimens and provide references for the selection of specimens in the diagnosis of LNTB.

Our research found that the pooled sensitivity and specificity of Xpert MTB/RIF for LNTB were 79\% (95\% CI 69-86\%) and 98\% (95\% CI 94-99\%) versus CRS and 

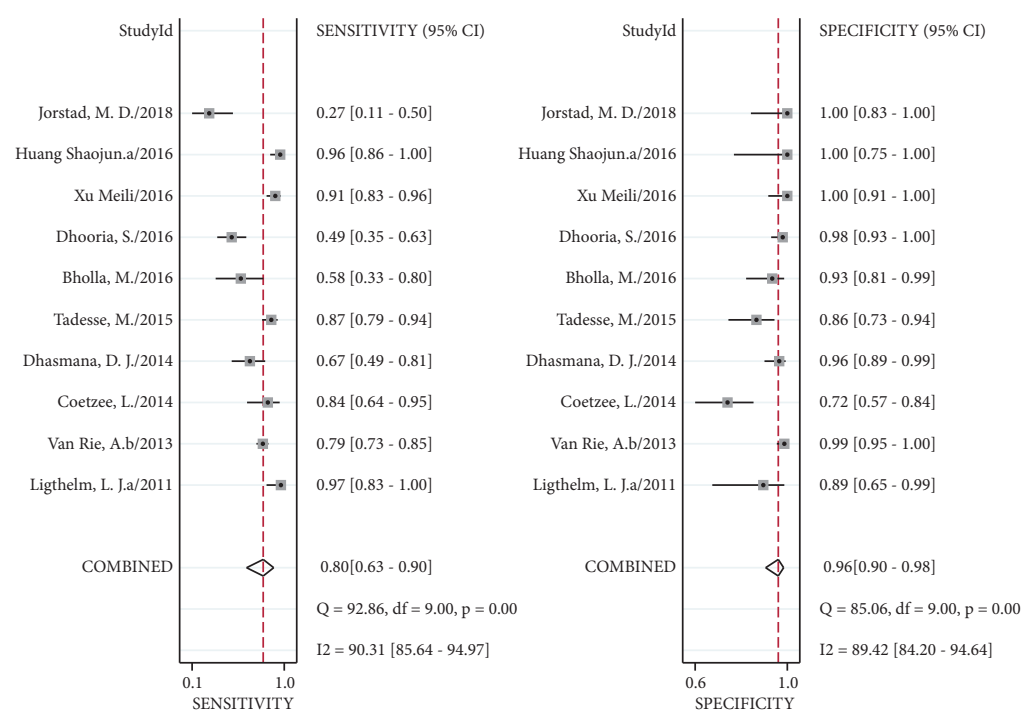

(a)
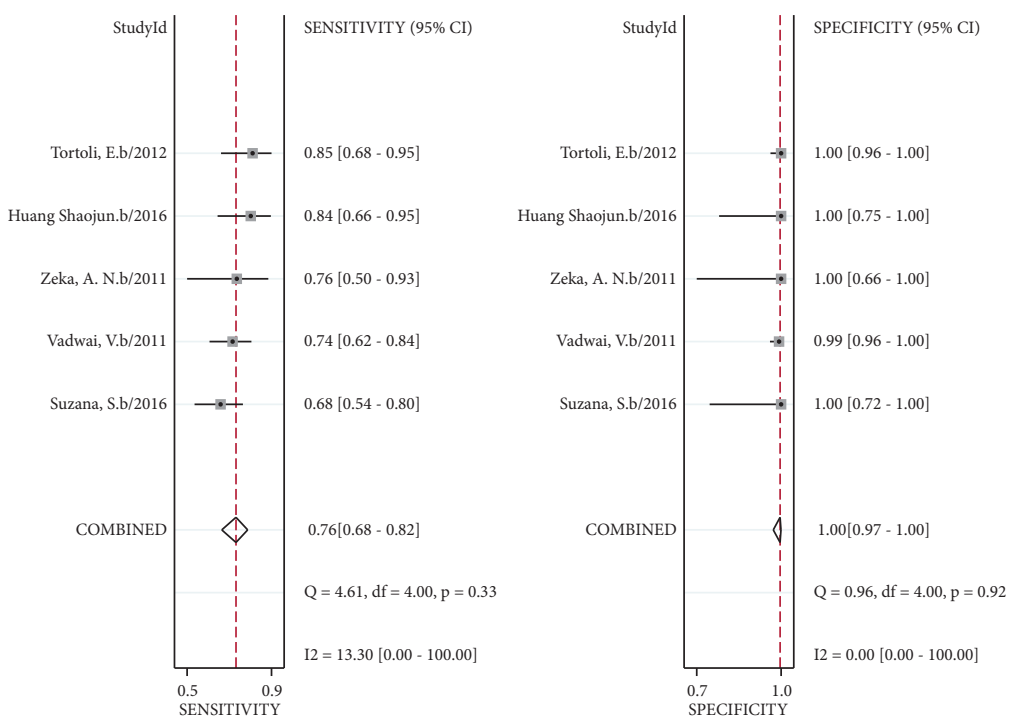

(b)

FIGURE 5: Forest plot of Xpert sensitivity and specificity for tuberculosis detection in LNTB versus composite reference standard. (a) FNA samples. (b) Tissue samples. The squares represent the sensitivity and specificity of a study, and the black line represents their confidence intervals. The diamonds represent the pooled sensitivity and specificity and their confidence intervals. LNTB: lymph nodes tuberculosis. FNA: fine needle aspiration.

84\% (95\% CI 77-90\%) and 91\% (95\% CI 78-96\%) versus culture, respectively. In a meta-analysis by Denkinger et al., Xpert MTB/RIF pooled sensitivity and specificity were $81.2 \%$ (95\% CI 72.4-87.7\%) and 99.1\% (95\% CI 94.5-99.9\%) versus CRS, and $83.1 \%$ (95\% CI 71.4-90.7\%) and 93.6\% (95\% CI 87.9-96.8\%) versus culture for lymph node tissues or aspirates [3]. The results were similar to our study; however, it failed to make further analysis based on the type of specimens. In the present study, significant heterogeneity was also observed among the studies. Subgroup analysis revealed the pooled sensitivity of Xpert MTB/RIF, performed on FNA samples, to be $80 \%$ (95\% CI 63-90\%) and 90\% (95\% CI 83-95\%) and the pooled specificity to be $96 \%$ (95\% CI 90-98\%) and 89\% (95\%
CI 65-97\%) compared to CRS and culture, respectively. The pooled sensitivity and specificity were 76\% (95\% CI 68-82\%) and $76 \%$ (95\% CI 66-83\%) and 100\% (95\% CI 97-100\%) and 92\% (95\% CI 78-98\%) compared to CRS and culture, when using tissue specimens. Metaregression analysis revealed that the sensitivity of Xpert MTB/RIF using FNA specimens was higher than that of using tissue specimens, regardless of the gold standard, although statistical difference was not significant. The sample reagent buffer used in the Xpert assay had originally been designed for the liquefaction of mucoid sputum samples. Tissues may not be completely homogenized with the same buffer, thus resulting in low sensitivity [30]; FNA specimens are more likely to be homogenized. 

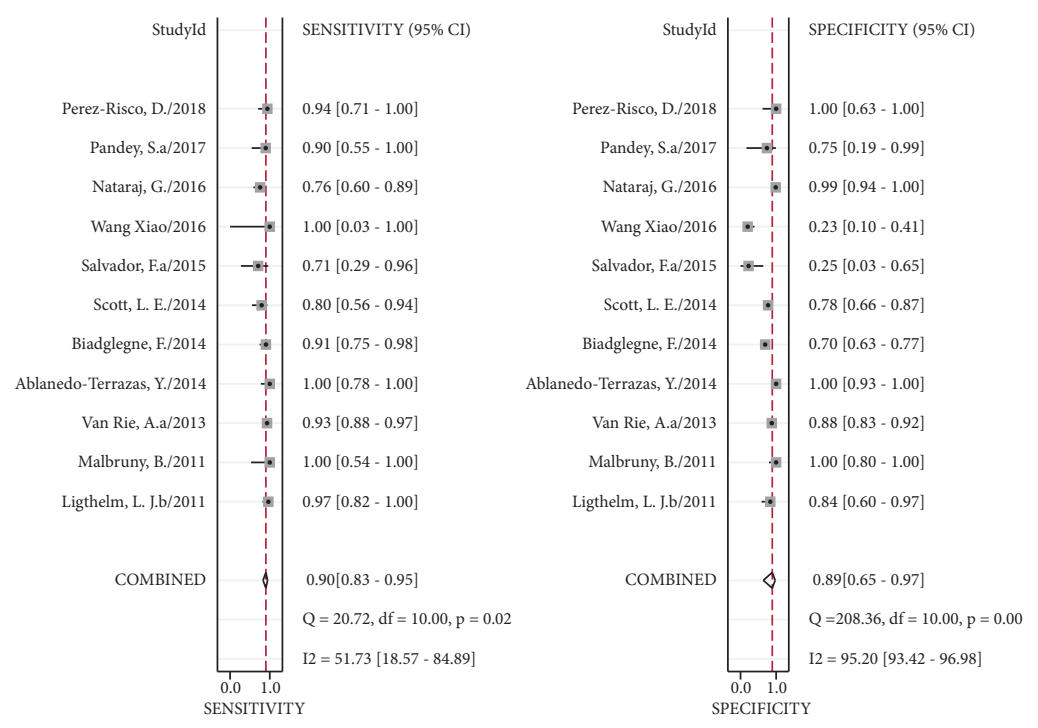

(a)
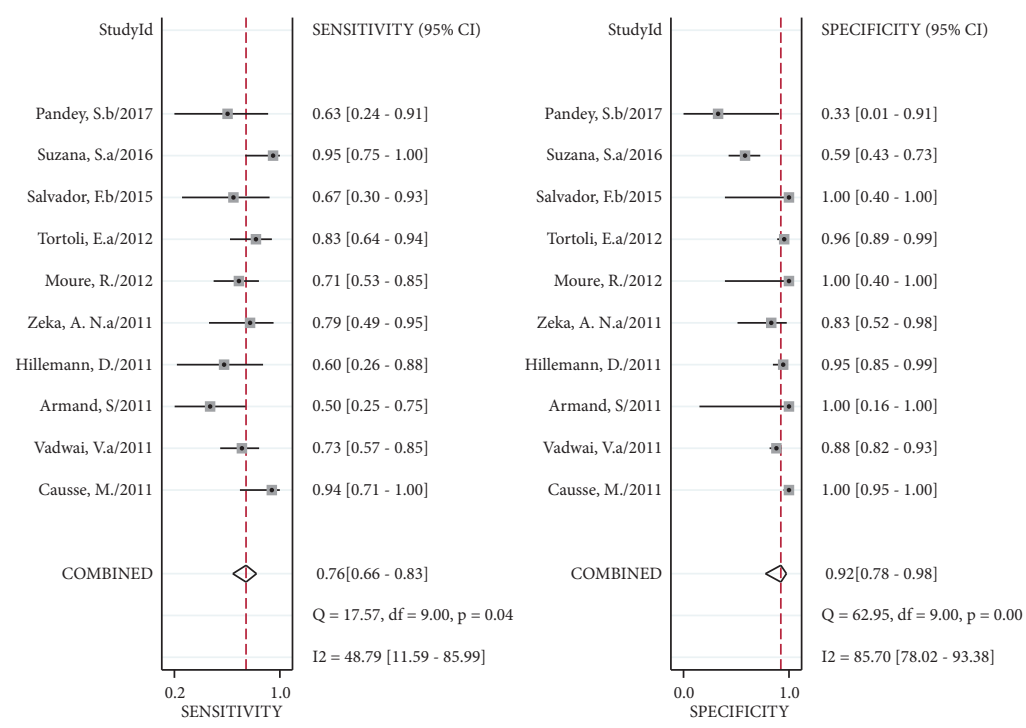

(b)

FIGURE 6: Forest plot of Xpert sensitivity and specificity for tuberculosis detection in LNTB versus culture. (a) FNA samples. (b) Tissue samples. The squares represent the sensitivity and specificity of a study, and the black line represents their confidence intervals. The diamonds represent the pooled sensitivity and specificity and their confidence intervals. LNTB: lymph nodes tuberculosis. FNA: fine needle aspiration.

This may possibly explain why FNA specimens have higher sensitivity than tissues. On the contrary, the specificity of Xpert MTB/RIF using FNA specimens was lower than that of using tissue specimens regardless of the gold standard; statistical difference was significant when considered against a CRS. Overall, the diagnostic efficiency of Xpert MTB/RIF for LNTB, using FNA and tissue specimens, was found to be similar. Owing to its paucibacillary nature, for LNTB, a CRS might be a more applicable reference standard. However, the CRS varied across studies in this research. CRS for all studies included the results of culture while that for most studies included the results of histology/cytology and smear microscopy; a few studies included the clinical features and radiology results, three studies included the response to antituberculosis treatment, and only one study included the result of Xpert MTB/RIF [19]. This might be one of the sources of heterogeneity across studies.

Sample processing of lymph node specimens, such as decontamination, sample condition, and homogenization, was variable across studies, but metaregression analysis showed that these factors did not affect the result and hence were not the sources of heterogeneity. In addition, we also found the patient's financial status to not affect the outcome.

According to the findings of this study, a multistep approach could be adopted for the management of suspected LNTB: FNA should be performed as the first step due to its less invasiveness, followed by complete relevant examinations, including Xpert MTB/RIF and pathological tests to 
improve diagnostic sensitivity. When the inspection result of the first step is negative, a more invasive technique (like biopsy) should be performed; pathological examination may be used for further validation, rather than Xpert MTB/RIF, since the assay did not increase the sensitivity of diagnosis when performed in biopsy-obtained samples.

Our meta-analysis also had several limitations. We realize that we may have missed some studies, despite the comprehensive search, and some studies that failed to distinguish specimen types. In addition, some included studies used multiple sample types, which may have led to some bias in our results. In addition, sample processing of lymph node specimens was highly variable across and within studies, since the assay, designed for respiratory samples, may slightly vary for other specimens. Additionally, the CRS standard for the studies was also different. Heterogeneity among the studies was remarkable, and the pooled estimates need to be interpreted with caution.

\section{Conclusions}

In this meta-analysis, we observed that the pooled sensitivity and specificity of Xpert MTB/RIF were $79 \%$ and $98 \%$, respectively, when compared with a CRS, and $84 \%$ and $91 \%$, respectively, when compared with culture. When performed on FNA samples, the pooled sensitivity and specificity were $80 \%$ and $96 \%$ versus CRS and $90 \%$ and $89 \%$ versus culture, respectively. When performed on tissue samples, the pooled sensitivity and specificity were $76 \%$ and $100 \%$ versus CRS, and $76 \%$ and $92 \%$ versus culture, respectively. There was no significant difference in the diagnostic efficiency for specimens obtained via different routes. Xpert MTB/RIF showed a good diagnostic efficiency on LNTB and was not related to the type of specimen.

\section{Conflicts of Interest}

The authors have no funding or conflicts of interest to disclose.

\section{Supplementary Materials}

Search strategies for each database. (Supplementary Materials)

\section{References}

[1] World Health Organization, "Global tuberculosis report," Tech. Rep., World Health Organization, 2016.

[2] L. Maynard-Smith, N. Larke, J. A. Peters, and S. D. Lawn, "Diagnostic accuracy of the Xpert MTB/RIF assay for extrapulmonary and pulmonary tuberculosis when testing nonrespiratory samples: a systematic review," BMC Infectious Diseases, vol. 14, no. 1, article 709, 2014.

[3] C. M. Denkinger, S. G. Schumacher, C. C. Boehme, N. Dendukuri, M. Pai, and K. R. Steingart, "Xpert MTB/RIF assay for the diagnosis of extrapulmonary tuberculosis: a systematic review and meta-analysis," European Respiratory Journal, vol. 44, no. 2, pp. 435-446, 2014.
[4] P. F. Whiting, A. W. S. Rutjes, M. E. Westwood et al., "Quadas-2: a revised tool for the quality assessment of diagnostic accuracy studies," Annals of Internal Medicine, vol. 155, no. 8, pp. 529-536, 2011.

[5] P. Macaskill, C. Gatsonis, J. Deeks, R. M. Harbord, and Y. Takwoingi, Cochrane handbook for systematic reviews of diagnostic test accuracy version 1.0, The Cochrane collaboration, 2010, http://srdta.cochrane.org/.

[6] J. P. T. Higgins, S. G. Thompson, J. J. Deeks, and D. G. Altman, "Measuring inconsistency in meta-analyses," British Medical Journal, vol. 327, no. 7414, pp. 557-560, 2003.

[7] Y. Zhou and N. Dendukuri, "Statistics for quantifying heterogeneity in univariate and bivariate meta-analyses of binary data: the case of meta-analyses of diagnostic accuracy," Statistics in Medicine, vol. 33, no. 16, pp. 2701-2717, 2014.

[8] J. B. Reitsma, A. W. S. Rutjes, K. S. Khan, A. Coomarasamy, and P. M. Bossuyt, "A review of solutions for diagnostic accuracy studies with an imperfect or missing reference standard," Journal of Clinical Epidemiology, vol. 62, no. 8, pp. 797-806, 2009.

[9] I. Schiller, M. van Smeden, A. Hadgu, M. Libman, J. B. Reitsma, and N. Dendukuri, "Bias due to composite reference standards in diagnostic accuracy studies," Statistics in Medicine, vol. 35, no. 9, pp. 1454-1470, 2016.

[10] C. A. Naaktgeboren, L. C. Bertens, M. van Smeden, J. A. de Groot, K. G. Moons, and J. B. Reitsma, "Value of composite reference standards in diagnostic research," British Medical Journal, vol. 347, article f5605, 2013.

[11] S. Armand, P. Vanhuls, G. Delcroix, R. Courcol, and N. Lemaitre, "Comparison of the Xpert MTB/RIF test with an IS6110-TaqMan real-time PCR assay for direct detection of Mycobacterium tuberculosis in respiratory and nonrespiratory specimens," Journal of Clinical Microbiology, vol. 49, no. 5, pp. 1772-1776, 2011.

[12] M. Causse, P. Ruiz, J. B. Gutiérrez-Aroca, and M. Casal, "Comparison of two molecular methods for rapid diagnosis of extrapulmonary tuberculosis," Journal of Clinical Microbiology, vol. 49, no. 8, pp. 3065-3067, 2011.

[13] D. Hillemann, S. Rüsch-Gerdes, C. Boehme, and E. Richter, "Rapid molecular detection of extrapulmonary tuberculosis by the automated genexpert MTB/RIF system," Journal of Clinical Microbiology, vol. 49, no. 4, pp. 1202-1205, 2011.

[14] L. J. Ligthelm, M. P. Nicol, K. G. P. Hoek et al., "Xpert MTB/RIF for rapid diagnosis of tuberculous lymphadenitis from fineneedle-aspiration biopsy specimens," Journal of Clinical Microbiology, vol. 49, no. 11, pp. 3967-3970, 2011.

[15] B. Malbruny, G. Le Marrec, K. Courageux, R. Leclercq, and V. Cattoir, "Rapid and efficient detection of Mycobacterium tuberculosis in respiratory and non-respiratory samples," The International Journal of Tuberculosis and Lung Disease, vol. 15, no. 4, pp. 553-555, 2011.

[16] V. Vadwai, C. Boehme, P. Nabeta, A. Shetty, D. Alland, and C. Rodrigues, "Xpert MTB/RIF: a new pillar in diagnosis of extrapulmonary tuberculosis?" Journal of Clinical Microbiology, vol. 49, no. 7, pp. 2540-2545, 2011.

[17] A. N. Zeka, S. Tasbakan, and C. Cavusoglu, "Evaluation of the GeneXpert MTB/RIF assay for rapid diagnosis of tuberculosis and detection of rifampin resistance in pulmonary and extrapulmonary specimens," Journal of Clinical Microbiology, vol. 49, no. 12, pp. 4138-4141, 2011.

[18] R. Moure, R. Martín, and F. Alcaide, "Effectiveness of an integrated real-time PCR method for detection of the 
Mycobacterium tuberculosis complex in smear-negative extrapulmonary samples in an area of low tuberculosis prevalence," Journal of Clinical Microbiology, vol. 50, no. 2, pp. 513-515, 2012.

[19] E. Tortoli, C. Russo, C. Piersimoni et al., "Clinical validation of Xpert MTB/RIF for the diagnosis of extrapulmonary tuberculosis," European Respiratory Journal, vol. 40, no. 2, pp. 442-447, 2012.

[20] A. Van Rie, L. Page-Shipp, K. Mellet et al., "Diagnostic accuracy and effectiveness of the Xpert MTB/RIF assay for the diagnosis of HIV-associated lymph node tuberculosis," European Journal of Clinical Microbiology \& Infectious Diseases, vol. 32, no. 11, pp. 1409-1415, 2013.

[21] Y. Ablanedo-Terrazas, C. Alvarado-De La Barrera, R. Hernández-Juan, M. Ruiz-Cruz, and G. Reyes-Terán, "Xpert MTB/RIF for diagnosis of tuberculous cervical lymphadenitis in HIV-infected patients," The Laryngoscope, vol. 124, no. 6, pp. 1382-1385, 2014.

[22] F. Biadglegne, A. Mulu, A. C. Rodloff, and U. Sack, "Diagnostic performance of the Xpert MTB/RIF assay for tuberculous lymphadenitis on fine needle aspirates from Ethiopia," Tuberculosis, vol. 94, no. 5, pp. 502-505, 2014.

[23] L. Coetzee, M. P. Nicol, R. Jacobson et al., "Rapid diagnosis of pediatric mycobacterial lymphadenitis using fine needle aspiration biopsy," The Pediatric Infectious Disease Journal, vol. 33, no. 9, pp. 893-896, 2014.

[24] D. J. Dhasmana, C. Ross, C. J. Bradley et al., "Performance of Xpert MTB/RIF in the diagnosis of tuberculous mediastinal lymphadenopathy by endobronchial ultrasound," Annals of the American Thoracic Society, vol. 11, no. 3, pp. 392-396, 2014.

[25] L. E. Scott, N. Beylis, M. Nicol et al., "Diagnostic accuracy of xpert MTB/RIF for extrapulmonary tuberculosis specimens: Establishing a laboratory testing algorithm for South Africa," Journal of Clinical Microbiology, vol. 52, no. 6, pp. 1818-1823, 2014.

[26] F. Salvador, I. Los-Arcos, A. Sánchez-Montalvá et al., "Epidemiology and diagnosis of tuberculous lymphadenitis in a tuberculosis low-burden country," Medicine, vol. 94, no. 4, Article ID e509, 2015.

[27] M. Tadesse, G. Abebe, K. Abdissa et al., "GeneXpert MTB/RIF assay for the diagnosis of tuberculous lymphadenitis on concentrated fine needle aspirates in high tuberculosis burden settings," PLoS ONE, vol. 10, Article ID e0137471, 2015.

[28] M. Bholla, N. Kapalata, E. Masika et al., "Evaluation of Xpert ${ }^{\circledR}$ MTB/RIF and Ustar EasyNAT ${ }^{\mathrm{TM}}$ TB IAD for diagnosis of tuberculous lymphadenitis of children in Tanzania: a prospective descriptive study," BMC Infectious Diseases, vol. 16, p. 246, 2016.

[29] S. Dhooria, N. Gupta, A. Bal et al., "Role of Xpert MTB/RIF in differentiating tuberculosis from sarcoidosis in patients with mediastinal lymphadenopathy undergoing EBUS-TBNA: a study of 147 patients," Sarcoidosis Vasculitis and Diffuse Lung Diseses, vol. 33, no. 3, pp. 258-266, 2016.

[30] G. Nataraj, S. Kanade, and P. Mehta, "XpertW MTB/RIF for improved case detection of extra-pulmonary TB in a tertiary care setting in urban India," The International Journal of Tuberculosis and Lung Disease, vol. 20, no. 7, pp. 890-894, 2016.

[31] S. Suzana, M. M. Ninan, M. Gowri, K. Venkatesh, P. Rupali, and J. S. Michael, "Xpert MTB/Rif for the diagnosis of extrapulmonary tuberculosis - an experience from a tertiary care centre in South India," Tropical Medicine \& International Health, vol. 21, no. 3, pp. 385-392, 2016.
[32] H. Shaojun, Application of Molecular Biological Diagnosis in Diagnosis of Lymph Node Tuberculosis, Beijing tuberculosis and Thoracic Tumor Research Institute, Beijing, China, 2016.

[33] W. Xiao, W. Qiang, Y. Jianbo et al., "Comparative study of GeneXpert Mtb/RIF, acid fast staining and culture in diagnosis of superficial lymph node tuberculosis," Journal of Zunyi Medical University, pp. 275-278, 2016 (Chinese).

[34] X. Meili, Diagnostic Value of Fine Needle Aspiration Gene Xpert MTB/RIF Test for Lymph Node Tuberculosis, Hebei Medical University, 2016.

[35] S. Pandey, J. Congdon, B. McInnes, A. Pop, and C. Coulter, "Evaluation of the GeneXpert MTB/RIF assay on extrapulmonary and respiratory samples other than sputum: a low burden country experience," Pathology, vol. 49, no. 1, pp. 70-74, 2017.

[36] M. D. Jørstad, M. Marijani, A. M. Dyrhol-Riise, L. Sviland, and T. Mustafa, "MPT64 antigen detection test improves routine diagnosis of extrapulmonary tuberculosis in a low-resource setting: A study from the tertiary care hospital in Zanzibar," PLoS ONE, vol. 13, Article ID e0196723, 2018.

[37] D. Perez-Risco, D. Rodriguez-Temporal, I. Valledor-Sanchez, and F. Alcaidea, "Evaluation of the Xpert MTB/RIF ultra assay for direct detection of mycobacterium tuberculosis complex in smear-negative extrapulmonary samples," Journal of Clinical Microbiology, vol. 56, no. 9, Article ID e00659-18, 2018.

[38] L. Scott E, N. Beylis, M. Nicol et al., "Diagnosing extra pulmonary tuberculosis using Xpert MTB/RIF: a laboratory algorithm," Topics in Antiviral Medicine, vol. 22, p. 424, 2014.

[39] Z. F. Chen, H. L. Lao, X. H. Li et al., "Experimental study of GeneXpert( $\left.{ }^{\circledR}\right)$ system in the diagnosis of extra-pulmonary tuberculosis," Chinese Journal of Tuberculosis and Respiratory Diseases, vol. 39, no. 7, pp. 529-533, 2016.

[40] C. Zifang, L. Haili, L. Xiuhua et al., "Application value of automatic medical PCR analysis system in diagnosis of extrapulmonary tuberculosis and rifampicin resistance detection," Chinese Journal of Tuberculosis and Respiratory Diseases, pp. 529-533, 2016 (Chinese).

[41] A. Jain, P. K. Singh, U. Singh, and V. Kumar, "Initial screening of extra-pulmonary tuberculosis using the Xpert MTB/RIF assay improves case detection rates," The International Journal of Tuberculosis and Lung Disease, vol. 21, no. 4, pp. 478-480, 2017.

[42] C. Q. Yang, X. Y. Liu, R. H. Du, T. Z. Cao, and X. Y. Dai, "Diagnosis value with Xpert Mtb/RIF assay for cervical tuberculous lymphadenitis," Lin Chung Er Bi Yan Hou Tou Jing Wai Ke Za Zhi, vol. 31, pp. 1338-1340, 2017.

[43] Y. Chenqing, D. Xiyong, L. Quan et al., "Clinical analysis of 210 cases of cervical lymph node tuberculosis," Chinese Journal of Antituberculosis, vol. 2, pp. 169-173, 2017 (Chinese).

[44] A. Polesky, W. Grove, and G. Bhatia, "Peripheral tuberculous lymphadenitis: epidemiology, diagnosis, treatment, and outcome," Medicine, vol. 84, no. 6, pp. 350-362, 2005.

[45] J.-M. Fontanilla, A. Barnes, and C. F. von Reyn, "Current diagnosis and management of peripheral tuberculous lymphadenitis," Clinical Infectious Diseases, vol. 53, no. 6, pp. 555$562,2011$.

[46] S. Greco, E. Girardi, A. Navarra, and C. Saltini, "Current evidence on diagnostic accuracy of commercially based nucleic acid amplification tests for the diagnosis of pulmonary tuberculosis," Thorax, vol. 61, no. 9, pp. 783-790, 2006.

[47] R. Brownell, J. Metcalfe, A. J. Millman et al., "Performance of Xpert MTB/RIF for diagnosis of pulmonary and 
extra-pulmonary tuberculosis-a systematic review and metaanalysis," American Journal of Respiratory and Critical Care Medicine, p. 185, 2012.

[48] E. Penz, J. Boffa, D. J. Roberts et al., "Diagnostic accuracy of the Xpert ${ }^{\circledR}$ MTB/RIF assay for extrapulmonary tuberculosis: a meta-analysis," The International Journal of Tuberculosis and Lung Disease, vol. 19, no. 3, pp. 278-284, 2015. 


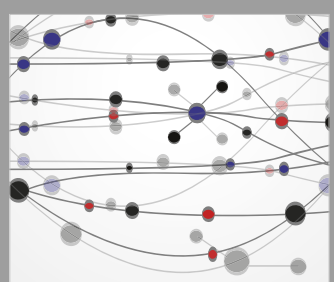

The Scientific World Journal
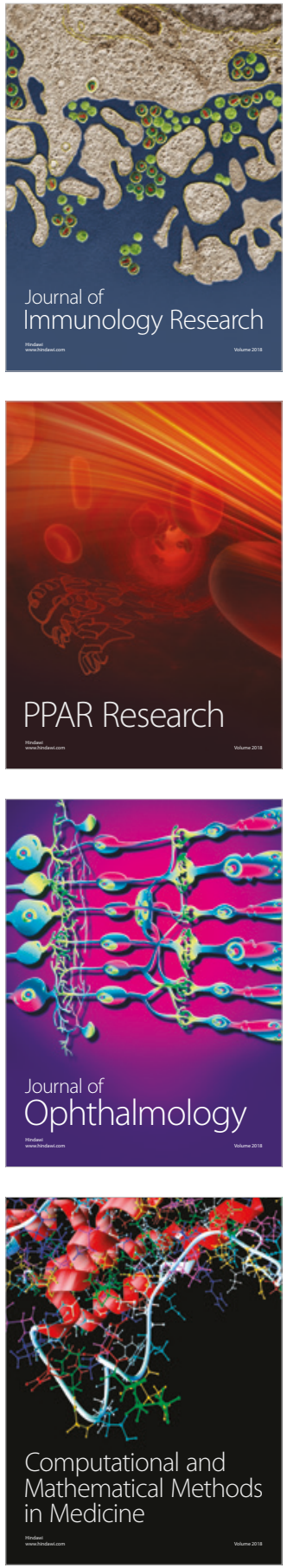

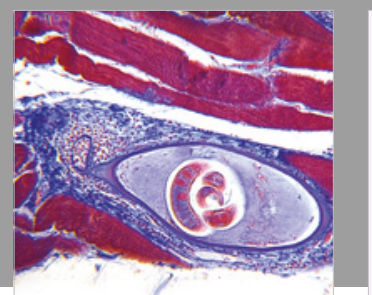

Gastroenterology Research and Practice

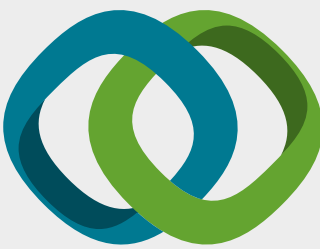

\section{Hindawi}

Submit your manuscripts at

www.hindawi.com
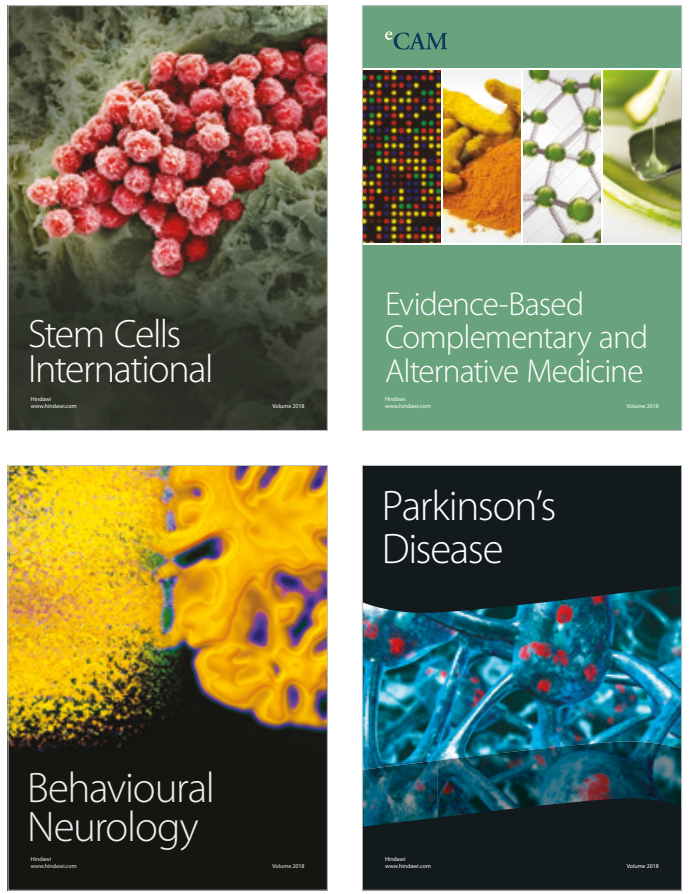

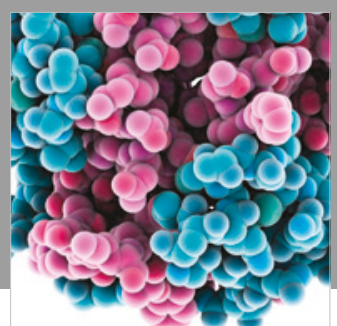

ournal of

Diabetes Research

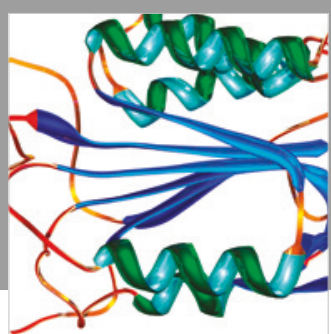

Disease Markers
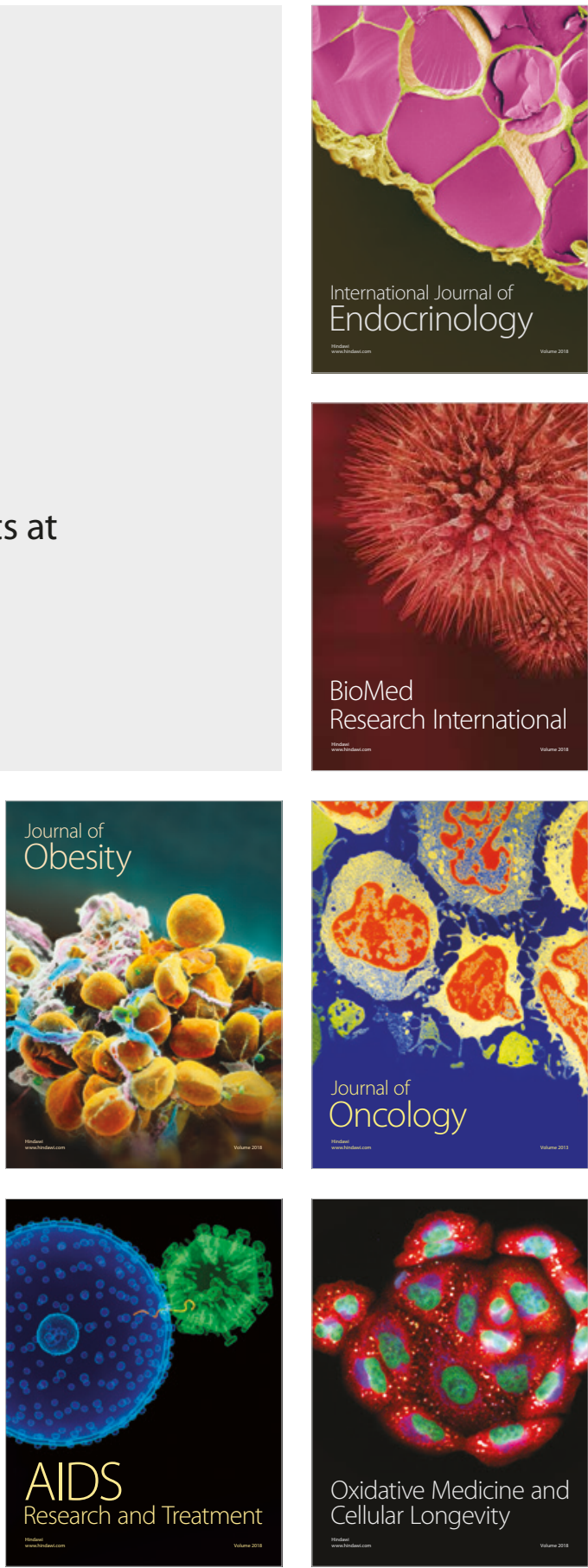